\title{
Ollie was Right! A Review of Angular Dependence, Detector Bandwidth and Sample Preparation on Contrast in Secondary and Backscattered Electron Images in the SEM.
}

\author{
B.J. Griffin ${ }^{1,2}$, D.C. Joy ${ }^{3}$ and J.R. Michael ${ }^{4}$
}

1 Centre for Forensic Science, The University of Western Australia, Crawley, WA Australia 6009

2 Centre for Microscopy, Characterization and Analysis, The University of Western Australia, Crawley, WA Australia 6009

3 Center for NanoPhase Materials Science, Oak Ridge National Laboratory, Oak Ridge, TN 37831

4 Sandia National Laboratories, PO Box 5800, Albuquerque, NM 87185-0886

Oliver Wells started SEM research in 1953 on the only existing instrument, in the Oatley group in Cambridge. From 1965 at IBM he continued this research into essentially the information content of image contrast in SEM. The 1960 Everhard-Thornley SED design dominated SE imaging and was recognized to capture a range of SE signals. However the efficiency, relative 'cleanliness', simplicity and low cost of these SED coupled with a rapidly developing user interface meant that the SEM was, by the 1980's, considered mature. Attention focused, largely, on TEM development.

Modern SEM have evolved significantly in the last decade. Current field emission SEM may contain up to 2-3 BSED and 3 SED in column and chamber configurations, plus STEM and CL detectors. Sample biasing has also re-emerged for high resolution and/or low energy imaging. A range of 'novel' contrasts have been reported, particularly in the area of 'low-loss BSEI'.

A comparison of the various BSED and SED on current SEM has been undertaken in this study. The aim was to confirm the early work of Oliver Wells on the effects of the various parameters on SE image contrasts, and the sample information such contrasts define. The samples included a carbon coated, polished resin mount of bauxite and an air dried Pt coated slater. Imaging of the Metroboost imaging sample was also undertaken together with alumina nanoparticles.

The images show a range of contrasts (figures 1-4) with BSED and SED mounted at different postions. The visibility of the surface contamination in the 'n-beam' SEI at high beam energy $(20 \mathrm{kV})$ through the carbon coating is very high. Channelling and Moire fringe effects also form part of the image contrasts and mask true sample features (figure 5-6).

\section{References}

[1] Images in figures 1-4 are courtesy of TESCAN ORSAY.

[2] Sandia is a multiprogram laboratory operated by Sandia Corporation, a Lockheed Martin Company, for the United States Department of Energy (DOE) under contract DE-AC0494AL85000. 


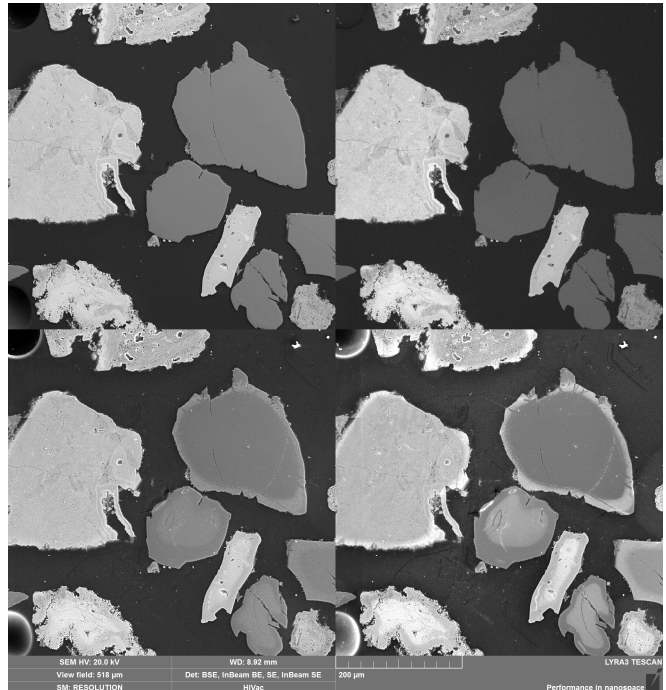

Figure 1: Comparison of the contrast between in-column and below-column $\operatorname{BSED}(a-b)$ and BSED (c-d) at $0{ }^{\circ}$ stage tilt and $20 \mathrm{kV}$ (bauxite sample).

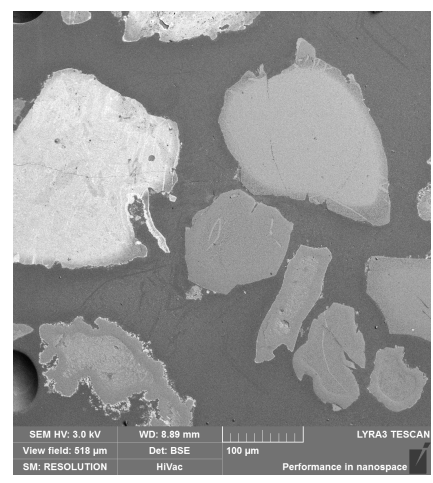

Figure 3: Below-column BSED image at $3 \mathrm{kV}$, and $0^{\circ}$ stage tilt(bauxite sample).

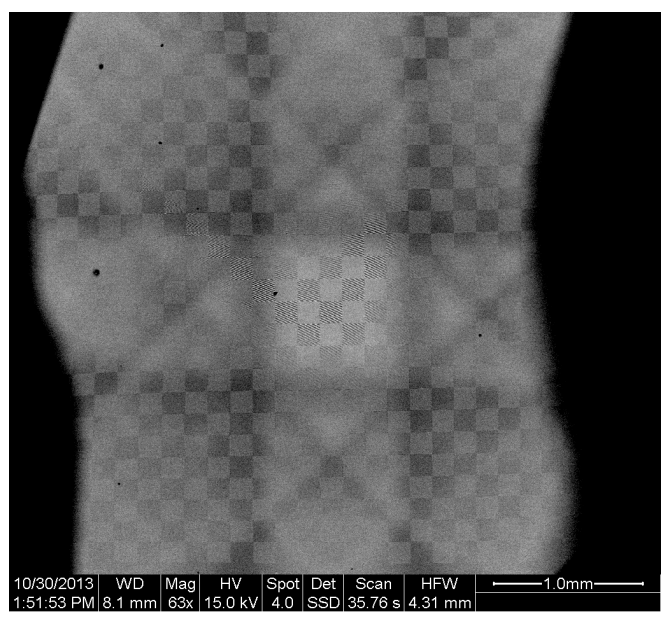

Figure 5: Image of $100 \mathrm{~nm}$ pitch squares on the. Metroboost sample. Channelling and Moire fringes are imposed on the sample information.

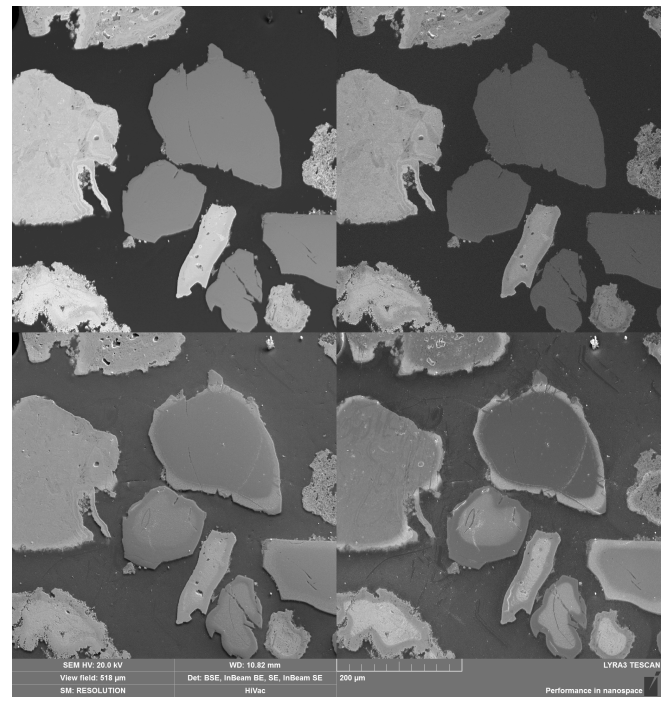

Figure 2: Comparison of the contrast between in-column and below-column BSED(a-b) and SED (c-d) at $55^{\circ}$ stage tilt and $20 \mathrm{kV}$ (bauxite sample).

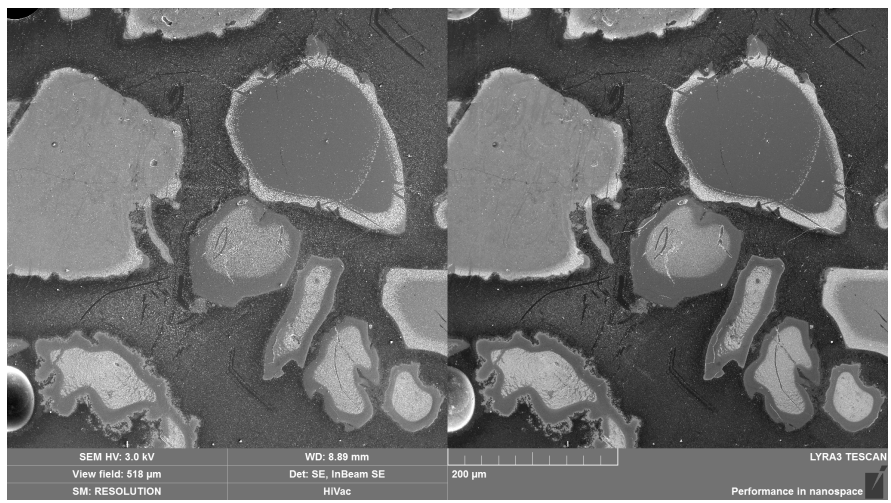

Figure 4: Below-column and above column SED images at $3 \mathrm{kV}$, and $0^{\circ}$ stage tilt (bauxite sample).

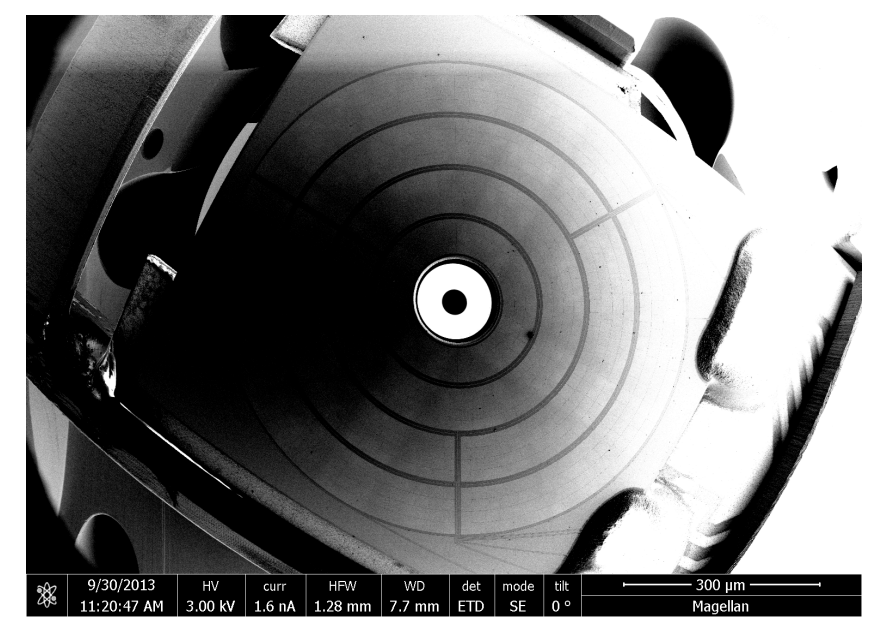

Figure 6: Electrostatic mirror image of a single crystal multi-element BSED on a FEI Magellan SEM. Channelling In the detector is evident. 